\title{
Performance of colposcopic scoring by modified International Federation of Cervical Pathology and Colposcopy terminology for diagnosing cervical intraepithelial neoplasia in a low-resource setting
}

\author{
Prabhakaran Nair Rema,Aleyamma Mathew', Shaji Thomas²
}

\begin{abstract}
Introduction: Colposcopy is a tool to evaluate women with cervical pre-cancer and cancer. To interpret the colposcopic findings, various scoring systems are used but with inter observer variations. To improve the quality of colposcopy, International Federation of Cervical Pathology and Colposcopy (IFCPC) has introduced a colposcopic nomenclature in 20I I. Colposcopic scoring helps to select patients who need treatment for cervical intraepithelial neoplasia. Aim of the Study:The study aimed to evaluate the agreement between colposcopic diagnosis with the modified IFCPC terminology and cervical pathology in patients with abnormal screening tests and to assess the utility of this colposcopic scoring system in low resource settings. Methodology: Patients with abnormal screening tests who underwent colposcopic assessment in the department of Gynaecological oncology were included in the study. Colposcopic scoring was done by the modified IFCPC nomenclature. The results were compared with cytology and the final histopathology. Results: 56 patients were included in the study. The colposcopic scoring when compared to histopathology showed agreement in $65.7 \%$ which indicated the agreement was substantial and was statistically significant $(P=0.000 \mathrm{I})$. With cytology the colposcopic score showed agreement in $35.6 \%$ indicating a fair agreement and this was also statistically significant $(P=0.00 \mathrm{I})$. Conclusion: Colposcopic scoring by modified IFCPC $20 \mathrm{II}$ criteria showed substantial agreement with cervical histopathology. Compared to traditional methods, 20II international terminology of colposcopy could improve colposcopic accuracy.
\end{abstract}

Key words: Cervical intraepithelial neoplasia, colposcopic scoring, colposcopy, International Federation of Cervical Pathology and Colposcopy nomenclature

\section{Introduction}

Cervical cancer still forms the major tumor burden of developing countries including India. Approximately 132,000 new cases and 74,000 deaths occur due to cervical cancer annually in India, accounting to nearly one-fifth of the global cervical cancer burden. ${ }^{[1]}$ It is estimated that cervical cancer will occur in approximately 1 in 53 Indian women during their lifetime compared with 1 in 100 women in developed countries. ${ }^{[2]}$ In developed countries, conventional cytology screening programs have shown a marked decline in the incidence of cervical cancer. Cervical cytology is the simplest and well-accepted screening test and has stood the test of time but has disadvantage of low sensitivity. Human papillomavirus DNA testing has more sensitivity but is costly and not uniformly available. Visual methods are the low-cost alternatives for low- and middle-income countries like India. ${ }^{[3]}$ Patients with abnormal screening tests are evaluated by colposcopy. Colposcopy identifies the lesion, aids-guided biopsy and helps to plan treatment.

Colposcopy is a real-time visualization of the cervix using a low-power microscope under magnification. The transformation zone (TZ) of ectocervix is inspected after applying acetic acid and Lugol's iodine for changes suggestive of cervical intraepithelial neoplasia (CIN), and biopsies are taken from these abnormal areas. Colposcopic evaluation should be done and documented in a precise and consistent way. As colposcopic findings depend on the skills of the colposcopist, there would be interobserver variations. To minimize the interobserver variations, many colposcopic scoring systems have been introduced. There are various colposcopic nomenclatures,

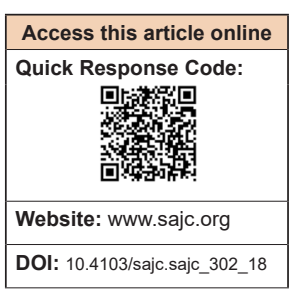

Division of Gynaecological Oncology, Regional Cancer Centre, 'Division of Epidemiology and Biostatistics, Regional Cancer Centre, ${ }^{2}$ Department of Surgical Services, Regional Cancer Centre, Trivandrum, Kerala, India

Correspondence to: Dr. Prabhakaran Nair Rema, E-mail:drremaanil@gmail.com but the latest and most popular one is the modified International Federation of Cervical Pathology and Colposcopy (IFCPC) nomenclature published in 2011. In the modified IFCPC nomenclature, the colposcopic findings are scored as normal, minor abnormality, major abnormality, suspicious of invasion, or miscellaneous findings. The new nomenclature has been validated in the developed countries, but the literature about its applicability in low-resource countries is scarce.

\section{Objectives}

i. To evaluate the agreement between colposcopic diagnosis with the modified IFCPC terminology and cervical pathology in patients with abnormal screening tests

ii. To assess the utility of the colposcopic scoring system in low-resource settings.

\section{Methodology}

This was a prospective study performed after the Institutional Review Board approval. The study population included patients aged between 21 and 55 who attended the colposcopic clinic from August 2017 to December 2017. These patients were referred with abnormal screening tests which included visual inspection with acetic acid (VIA) and cervical cytology. Exclusion criteria included pregnancy, posthysterectomy, and previous treatment for CIN. Informed consent was obtained from the patients. Colposcopy was done and scoring was performed using the modified IFCPC nomenclature. Colposcopic findings were described as normal, minor (Grade 1 abnormality), major (Grade 2 abnormality), or suspicious of invasion. Guided biopsies were taken from the abnormal areas.

This is an open access journal, and articles are distributed under the terms of the Creative Commons Attribution-NonCommercial-ShareAlike 4.0 License, which allows others to remix, tweak, and build upon the work non-commercially, as long as appropriate credit is given and the new creations are licensed under the identical terms.

For reprints contact: reprints@medknow.com

How to cite this article: Rema PN, Mathew A, Thomas S. Performance of colposcopic scoring by modified International Federation of Cervical Pathology and Colposcopy terminology for diagnosing cervical intraepithelial neoplasia in a low-resource setting. South Asian J Cancer 2019;8:218-20. 
Cytology findings were interpreted by the Bethesda system and histopathology as CIN. The treatment was given to the patient as per the institutional guidelines with observation and repeat cytology for patients with CIN1 and loop electrosurgical excision procedure (LEEP) for patients with CIN2 and CIN3.

Agreement between colposcopic diagnosis, cytology, and histopathology was assessed using Kappa statistics. Sensitivity, specificity, positive, and negative predictive values of the colposcopic scoring was estimated.

\section{Results}

Colposcopies were performed in 56 patients during the study. The mean age of the patients was 39.6 years (range: $21-55$ years). Three patients were postmenopausal. Forty-two patients were asymptomatic, while seven patients had irregular bleeding, one had postmenopausal bleeding, and five had abnormal vaginal discharge. Cytology results were available for 45 patients. Cytology was normal in eight patients who were referred with positive VIA. Cytology showed minor abnormality which included atypical squamous cells of undetermined significance (ASCUS) or low-grade squamous intraepithelial lesion (LSIL) in 12, high-grade squamous intraepithelial lesion in 23 , and squamous cell carcinoma in 2 patients [Table 1]. Colposcopic examination was adequate in 54 patients, and the squamocolumnar junction was visualized in 39 patients. Twenty-nine patients had Type 1, 19 had Type 2, and 8 had Type 3 TZs. The colposcopic score was normal in 16 patients, minor abnormality in 21 , major abnormality in 17 , and suspicious of invasion in 2 patients. The histopathological finding was normal in 15 patients, low-grade CIN in 19, high-grade CIN (CIN 2 and CIN 3) in 20 patients, and invasive carcinoma in 2 patients.

The measurement of agreement between colposcopic score and histopathology and colposcopic score and cytology was assessed with Cohen's Kappa coefficient, and the statistical significance was assessed using Chi-square test. The colposcopic scoring when compared to histopathology showed agreement in $65.7 \%$ which indicated the agreement was substantial and was statistically significant $(P=0.0001)$. With cytology, the colposcopic score showed agreement in $35.6 \%$ indicating a fair agreement, and this was also statistically significant $(P=0.001)$ [Table 2]. The diagnostic accuracy of colposcopic scoring in diagnosing CIN with histopathology as the gold standard was calculated. The colposcopic scoring had sensitivity of $100 \%$, specificity of $50 \%$, and the positive predictive value of the finding of any colposcopic abnormality for any histologic abnormality was $83.3 \%$.

\section{Discussion}

Colposcopy is considered a subjective procedure highly dependent on the skills of the individual colposcopist. To standardize the procedure and to minimize the interobserver variations many colposcopic scoring systems have been introduced. The scoring was based on the degree of acetowhitening of the lesion, the margins, vascular pattern, and the changes after applying Lugol's iodine. In 1985, Reid and Scalzi introduced the Reids colposcopic index (RCI) which was later modified as modified RCI..$^{[4,5]}$ The index considers colposcopic signs including lesion margin, color of
Table 1: Patient characteristics

\begin{tabular}{lc} 
Patient features & No of patients $N$ \\
\hline Age at diagnosis & \\
Mean & 39.6 years \\
Range & 21 -55 years \\
Symptoms & \\
Bleeding & 8 \\
Discharge & 5 \\
No symptoms & 42 \\
Cytology result & $n=45$ \\
Normal & 8 \\
Minor abnormality (ASCUS, LSIL) & 12 \\
Major abnormality (ASCH, HSIL) & 23 \\
Squamous cell carcinoma & 2 \\
Colposcopic score & $n=56$ \\
Normal & 16 \\
Minor abnormality & 21 \\
Major abnormality & 17 \\
Suspicious of invasion & 2 \\
Histopathology & $n=56$ \\
Normal & 15 \\
Low-grade CIN & 19 \\
High-grade CIN & 20 \\
Squamous cell carcinoma & 2 \\
\hline LSIL=Low-grade squamous intraepithelial lesion, HSIL=High-grade squamous \\
intraepithelial lesion, CIN=Cervical intraepithelial neoplasia, ASCUS=Atypical squamous \\
cells of undetermined significance, ASCH=Atypical squamous cells- cannot exclude HSIL
\end{tabular}

Table 2: Agreement between colposcopic score and pathology

\begin{tabular}{llc}
\hline Measure of agreement & к $(\%)$ & $P$ \\
\hline Colpo versus HPR $(n=56)$ & $\begin{array}{l}65.7 \text { (agreement is } \\
\text { substantial) }\end{array}$ & $0.0001^{*}$ \\
Colpo versus cytology $(n=45)$ & 35.6 (agreement is fair) & $0.001^{*}$ \\
\hline
\end{tabular}

*Statistically significant at $5 \%$ level. HPR=Histoppathology

acetowhitening, blood vessels, and iodine staining. Modified RCI was popular among colposcopists, but the results of ASCUS LSIL Triage Study showed poor correlation with histopathology and nonuniform scoring among colposcopists. This was followed by the Swede Score, proposed by Strander in 2005, which included the lesion size also but the correlation of Swede score also with histopathology was poor. $^{[6]}$

The IFCPC in 2011 proposed a revised colposcopic nomenclature including various normal and abnormal colposcopic findings. ${ }^{[7]}$ This nomenclature gives a description of colposcopic features which improves its accuracy over the colposcopic indices. The popular terms "satisfactory colposcopy" and "unsatisfactory colposcopy" have been replaced. It provides a general assessment in colposcopy including adequacy of colposcopy, visibility of squamocolumnar junction, and type of TZ. It has detailed the characteristics of lesions allowing classification as major and minor lesions and also has introduced two new colposcopic signs - the inner border and ridge signs. The following definitions have been added: congenital TZ, polyp (ectocervical or endocervical), stenosis, congenital anomaly, and posttreatment consequence. In addition, the terminology includes standardization of cervical excision treatment types and cervical excision specimen dimensions. The IFCPC recommends that the 2011 terminology 
replaces all others and be implemented for diagnosis, treatment, and research.

In the present study, we assessed the diagnostic sensitivity of the revised IFCPC criteria in diagnosing CIN with histopathology as the gold standard. We found the association between the colposcopic impression and histopathology was highly significant $(P<0.001)$ and the $\mathrm{k}$ value for the strength of correlation was $65.7 \%$ indicating good agreement. Li et al. in a retrospective study of 376 patients compared the agreement of colposcopic nomenclature by IFCPC terminology with histopathology by cervical biopsy, or conization found that agreement of colposcopic diagnosis and cervical pathology was $60.9 \%$ (229/376) perfectly matched, and the strength of agreement with weighted Kappa statistic was $0.401(P<0.01)$ comparable to our results. ${ }^{[8]}$ Tatiyachonwiphut et al. reported an exact agreement of 57.9 which was comparable to the results of our study. However, the study did not use the IFCPC nomenclature. The agreement with cytology was also significant in our study. ${ }^{[9]}$ Benedet et al. in a large study of 84,244 patients found that colposcopic impression correlates closely with the cytology diagnosis and combining the two produced optimum results. ${ }^{[10]}$ Compared to previous studies, the agreement between colposcopy and histopathology was higher in those following the IFCPC nomenclature. ${ }^{[8,11-14]}$

The present study showed a higher sensitivity and similar specificity compared to previous studies for colposcopy in diagnosing CIN by Baum et al. and Massad et al., but our study is limited by the smaller number of patients. ${ }^{[15,16]}$ Prospective studies including larger number of patients are needed to confirm the diagnostic accuracy of the revised IFCPC nomenclature. The relevant finding in our study is once the diagnostic accuracy of the revised IFCPC nomenclature has been proven therapeutic procedures such as LEEP or cryotherapy can be administered in the same sitting as diagnostic colposcopy as "see and treat protocol" avoiding cervical biopsy. This is highly applicable in a low-resource country such as India with a large burden of cervical cancer cases.

\section{Conclusion}

Colposcopic scoring by modified IFCPC 2011 criteria showed substantial agreement with cervical cytology and histopathology and could improve diagnostic accuracy of colposcopy

Financial support and sponsorship

Nil.

\section{Conflicts of interest}

There are no conflicts of interest.

\section{References}

1. Ferlay J, Soerjomataram I, Ervik M, Forman D, Bray F, Dixit R, et al. GLOBOCAN 2012, Cancer Incidence and Mortality Worldwide in 2012. Lyon, France: International Agency for Research on Cancer; 2012.

2. IInstitute for Health Metrics and Evaluation. The Challenge Ahead: Progress and setbacks in breast and cervical cancer. Seattle, WA: IHME, 2011.

3. Sankaranarayanan R, Nessa A, Esmy PO, Dangou JM. Visual inspection methods for cervical cancer prevention. Best Pract Res Clin Obstet Gynaecol 2012;26:221-32.

4. Reid R, Scalzi P. Genital warts and cervical cancer. VII. An improved colposcopic index for differentiating benign papillomaviral infections from high-grade cervical intraepithelial neoplasia. Am J Obstet Gynecol 1985; 153:611-8.

5. Ferris DG, Litaker MS; ALTS Group. Prediction of cervical histologic results using an abbreviated reid colposcopic index during ALTS. Am J Obstet Gynecol 2006;194:704-10.

6. Strander B, Ellström-Andersson A, Franzén S, Milsom I, Rådberg T. The performance of a new scoring system for colposcopy in detecting high-grade dysplasia in the uterine cervix. Acta Obstet Gynecol Scand 2005;84:1013-7.

7. Bornstein J, Bentley J, Bösze P, Girardi F, Haefner H, Menton M, et al. 2011 colposcopic terminology of the International Federation for Cervical Pathology and Colposcopy. Obstet Gynecol 2012;120:166-72.

8. Li Y, Zhang H, Zheng R, Xie F, Sui L. Agreement between colposcopic diagnosis with 2011 international terminology of colposcopy and cervical pathology in cervical lesions. Zhonghua Fu Chan Ke Za Zhi 2015;50:361-6.

9. Tatiyachonwiphut M, Jaishuen A, Sangkarat S, Laiwejpithaya S, Wongtiraporn W, Inthasorn P, et al. Agreement between colposcopic diagnosis and cervical pathology: Siriraj hospital experience. Asian Pac J Cancer Prev 2014; 15:423-6.

10. Benedet JL, Matisic JP, Bertrand MA. An analysis of 84244 patients from the British Columbia cytology-colposcopy program. Gynecol Oncol 2004;92:127-34.

11. Hammes LS, Naud P, Passos EP, Matos J, Brouwers K, Rivoire W, et al. Value of the international federation for cervical pathology and colposcopy (IFCPC) terminology in predicting cervical disease. J Low Genit Tract Dis 2007; 11:158-65.

12. Luyten A, Buttmann-Schweiger N, Hagemann I, Scherbring S, Boehmer G, Gieseking F, et al. Utility and reproducibility of the international federation for cervical pathology and colposcopy classification of transformation zones in daily practice: A multicenter study of the German colposcopy network. J Low Genit Tract Dis 2015;19:185-8.

13. Cristiani P, Costa S, Schincaglia P, Garutti P, de Bianchi PS, Naldoni C, et al. An online quality assurance program for colposcopy in a population-based cervical screening setting in Italy: Results on colposcopic impression. J Low Genit Tract Dis 2014;18:309-13.

14. Ghosh I, Mittal S, Banerjee D, Singh P, Dasgupta S, Chatterjee S, et al. Study of accuracy of colposcopy in VIA and HPV detection-based cervical cancer screening program. Aust N Z J Obstet Gynaecol 2014;54:570-5.

15. Baum ME, Rader JS, Gibb RK, McAlister RP, Powell MA, Mutch DG, et al. Colposcopic accuracy of obstetrics and gynecology residents. Gynecol Oncol 2006; 103:966-70.

16. Massad LS, Collins YC. Strength of correlations between colposcopic impression and biopsy histology. Gynecol Oncol 2003;89:424-8.

\section{Second Opinion Service by five cancer experts}

Are you frustrated with patients seeking opinion in higher center?

Do you feel much time and money is lost in patients travelling to metro for second opinion?

Here is the solution of getting consolidated opinion from five cancer experts for your patient - without him having to leave your city. www.cancerexpert.in can help you

Ongoing clinical trials in oncology in India

Do you want to get updated about clinical trials ongoing in India? WhatsApp to 9869425694 to be included in the discussion group

If you are interested in joining WhatsApp discussion about medico legal aspects, please send request to 9869425694 